Cardiometabolic Safety of Lumateperone (ITI-007): Post Hoc Analyses of Short-Term Randomized Trials and an OpenLabel Long-Term Study in Schizophrenia

\author{
John B. Edwards, $\mathrm{MD}^{1}$, Andrew Satlin, $\mathrm{MD}^{1}$, \\ Suresh Durgam, $\mathrm{MD}^{1}$, Robert E. Davis, $\mathrm{PhD}^{1}$, \\ Richard Chen, $\mathrm{PhD}^{1}$, Sharon Mates, $\mathrm{PhD}^{1}$ and \\ Christoph U. Correll, MD ${ }^{2}$
}

\begin{abstract}
${ }^{1}$ Intra-Cellular Therapies, Inc, New York, NY, USA, and ${ }^{2}$ The Zucker Hillside Hospital, Department of Psychiatry, Northwell Health, Glen Oaks, NY, USA; Hofstra Northwell School of Medicine, Department of Psychiatry and Molecular Medicine, Hempstead, NY, USA; and Charité Universitat Medizin, Department of Child and Adolescent Psychiatry, Berlin, Germany
\end{abstract}

Presenting Author: John B. Edwards

\begin{abstract}
Study Objective. Current treatments for schizophrenia are often associated with increased rates of metabolic syndrome (MetSy). MetSy is defined as meeting 3 of the following 5 criteria: waist circumference $>40$ in (men) or $>35$ in (women), triglycerides $=150 \mathrm{mg} / \mathrm{dL}$, high density lipoprotein cholesterol (HDL) $<40 \mathrm{mg} / \mathrm{dL}$ (men) or $<50 \mathrm{mg} / \mathrm{dL}$ (women), systolic blood pressure (BP) $=130 \mathrm{mmHg}$ or diastolic $\mathrm{BP}=85 \mathrm{mmHg}$, fasting glucose $=100 \mathrm{mg} / \mathrm{dL}$. Patients with MetSy have an elevated risk of developing type II diabetes and increased mortality due to cardiovascular disease. Lumateperone (lumateperone tosylate, ITI-007), a mechanistically novel antipsychotic that simultaneously modulates serotonin, dopamine, and glutamate neurotransmission, is FDA approved for the treatment of schizophrenia. This distinct pharmacological profile has been associated with favorable tolerability and a low risk of adverse metabolic effects in clinical trials. This post hoc analysis of 2 randomized, double-blind, placebocontrolled studies of patients with an acute exacerbation of schizophrenia compared rates of MetSy with lumateperone and risperidone. Data from an open-label long-term trial of lumateperone were also evaluated.

Method. The incidence and shift in MetSy were analyzed in data pooled from 2 short-term (4 or 6 week) placebo- and activecontrolled (risperidone $4 \mathrm{mg}$ ) studies of lumateperone $42 \mathrm{mg}$ (Studies 005 and 302). The pooled lumateperone data were compared with data for risperidone. Data from an open-label 1-year trial (Study 303) evaluated MetSy in patients with stable schizophrenia switched from prior antipsychotic (PA) treatment to lumateperone $42 \mathrm{mg}$.

Results. In the acute studies ( $n=256$ lumateperone $42 \mathrm{mg}, \mathrm{n}=255$ risperidone $4 \mathrm{mg}$ ), rates of MetSy were similar between groups at baseline (16\% lumateperone, $19 \%$ risperidone). At the end of treatment (EOT), MetSy was less common with lumateperone than with risperidone ( $13 \%$ vs $25 \%$ ). More lumateperone patients (46\%) compared with risperidone (25\%) patients improved from
\end{abstract}

having MetSy at baseline to no longer meeting MetSy criteria at EOT. Conversely, more patients on risperidone than on lumateperone developed MetSy during treatment (13\% vs 5\%). Differences in MetSy conversion rates were driven by changes in triglycerides and glucose. In the long-term study ( $n=602$ lumateperone $42 \mathrm{mg}$ ), $33 \%$ of patients had MetSy at PA baseline. Thirtysix percent of patients (36\%) with MetSy at PA baseline improved to no longer meeting criteria at EOT. Fewer than half that percentage shifted from not meeting MetSy criteria to having MetSy (15\%).

Conclusions. In this post hoc analysis, lumateperone $42 \mathrm{mg}$ patients had reduced rates of MetSy compared with risperidone patients. In the long-term study, patients with MetSy on PA switched to lumateperone $42 \mathrm{mg}$ had a reduction in the risk of MetSy. These results suggest that lumateperone $42 \mathrm{mg}$ is a promising new treatment for schizophrenia with a favorable metabolic profile. Funding. Intra-Cellular Therapies, Inc.

\section{Knowledge of The Recognition and Management of Tardive Dyskinesia Markedly Improved Among Psychiatrists: Assessing the Impact of Online Medical Education}

\author{
Thomas Finnegan, $\mathrm{PhD}^{1}$, Anjali Mehra, $\mathrm{MD}^{1}$, \\ Jovana Lubarda, $\mathrm{PhD}^{1}$, Chirag Shah, $\mathrm{PharmD}^{2}$ and \\ Andrew J. Cutler, MD ${ }^{3}$
}

${ }^{1}$ Medscape Education, New York, NY, USA, ${ }^{2}$ Neurocrine Bioscience, Inc, San Diego, CA, USA, and ${ }^{3}$ Neuroscience Education Institute, Chief Medical Officer, Carlsbad, CA, USA and SUNY Upstate Medical University, Clinical Associate Professor of Psychiatry, Syracuse, NY, USA

Presenting Author: Thomas Finnegan

\begin{abstract}
Introduction. Tardive dyskinesia (TD) is a persistent and potentially disabling movement disorder associated with prolonged exposure to antipsychotic medications that jeopardizes adherence to treatment and reduces quality of life. The recognition and management of TD can be challenging in many instances. An online activity was developed to assess the ability of continuing medical education (CME) to improve awareness of the recognition and management of TD among psychiatrists.

Methods. The online CME activity consisted of a 30-minute video discussion between three expert faculty. Educational effect was assessed by comparing a matched sample of psychiatrists' responses to four identical questions pre- and post-activity. A chisquare test identified significant differences between pre- and post-assessment responses. Cramer's V was used to calculate the effect size of the online education ( $\geq 0.16$ is considerable). Data were collected between June 26 and August 6, 2019.

Results. Activity participation resulted in a considerable educational effect among psychiatrists $(\mathrm{n}=739 ; \mathrm{V}=0.25, \mathrm{P}<0.001)$. The following areas showed significant $(\mathrm{P}<0.05)$ pre- vs post-
\end{abstract}


educational improvements: recognition of incidence of TD associated with different antipsychotic therapies, differentiation of TD from parkinsonism, and the personalized selection of therapies for the management of TD. $37 \%$ of psychiatrists had a measurable increase in confidence in understanding the role of the interprofessional team in recognizing TD after activity participation.

Conclusions. The results indicated that a CME-certified 30-minute video activity was effective at improving knowledge among psychiatrists for the recognition and management of TD. Future education should continue to address best practices in the care of patients with TD.

Funding. Neurocrine Bioscience, Inc

\section{Characterization and Treatment Goals of Patients on Long-Acting Injectable vs Oral Antipsychotics: Results from a Patient/Caregiver/ Psychiatrist Survey}

Heather M. Fitzgerald, PharmD ${ }^{1}$, Jason Shepherd, BA $^{2}$, Hollie Bailey, $\mathrm{BSc}^{2}$, Mia Berry, $\mathrm{BSc}^{2}$, Jack Wright, $\mathrm{MSc}^{2}$ and Maxine Chen, $\mathrm{PhD}^{3}$

${ }^{1}$ Lundbeck LLC, Deerfield, IL, USA, ${ }^{2}$ Adelphi Real World, Bollington, Cheshire, United Kingdom, and ${ }^{3}$ Otsuka Pharmaceutical Development \& Commercialization, Inc., Princeton, NJ, USA

Presenting Author: Heather M. Fitzgerald

\section{Abstract}

Background. Patient preferences in schizophrenia (SCZ), including identification of key goals and outcomes for treatment and relative importance of certain treatment goals to patients, have been assessed by several studies. However, there continues to be a lack of sufficient evidence on US patient attitudes and perceptions towards treatment goals and pharmacotherapy options in SCZ, especially taking into context long-acting injectable antipsychotics (LAIs) in this disease area. This lack of evidence is further pronounced in caregivers of individuals with SCZ. The objective of this analysis was to characterize patients with SCZ on LAIs vs patients on oral antipsychotics (OAPs) and evaluate the treatment goals of patients in each group.

Methods. This was a real-world, cross-sectional survey of US psychiatrists, patients $=18$ years old with a diagnosis of SCZ, and caregivers. Data was collected using the Disease Specific Programme (DSP) methodology, which has been previously published. Psychiatrists $(n=120)$ completed detailed record forms for next 8 consecutive outpatients and 2 inpatients matching inclusion criteria, including non-interventional clinical and subjective assessments. The same patients and their caregivers, if present, were invited by their psychiatrist to voluntarily complete a separate survey.

Results. Of 1135 patients on treatment where the physician provided survey data; 251 were on an LAI, and 884 were on an OAP. Mean (SD) time to SCZ diagnosis for those on an LAI was 10.3 (12.0) years vs 7.8 (10.5) years for those on OAPs. More patients in the LAI vs OAP group were being treated as an inpatient $(27.1 \%$ vs $15.7 \%$, respectively; $\mathrm{p}<0.0001$ ). Patients on an LAI reported being on their current medication regimen for less time (mean 1.7 years) vs those on OAPs (mean 2.5 years) ( $\mathrm{p}=0.0093)$. More patients on LAIs were unemployed due to disability vs those on OAPs (56.1\% vs $39.5 \%$, respectively), and less patients on LAIs were able to work part-time or full-time ( $21.1 \%$ or $4.1 \%)$ vs those on OAPs $(23.2 \%$ or $11.4 \%)$. More patients on an LAI had a caregiver vs those on OAPs ( $37.3 \%$ vs $26.1 \%$, respectively; $\mathrm{p}=0.0011)$. Regarding the most important treatment goals reported by patients, both groups reported similar preferences for decrease in disease symptoms (62\% on LAI vs $65 \%$ on OAPs) and thinking more clearly (53\% on LAI vs $46 \%$ on OAPs); however, a numerically higher proportion of LAI patients reported that the current medication helped decrease hospitalizations due to relapse vs those on OAPs ( $38 \%$ vs $32 \%$, respectively).

Discussion. Given the characteristics of patients participating in this real-world survey, those on LAIs exhibited qualities which indicate a higher severity of illness vs those on OAPs. Results suggest that treatment with LAIs is still mainly being provided to patients later in the disease course and/or who have adherence problems, despite a growing body of evidence of utility in younger patients earlier in the course of illness.

Funding. Otsuka Pharmaceutical Development \& Commercialization, Inc. and Lundbeck LLC

\section{A Novel Real-Time PCR Assay for Detection of HLA-A*31:01 in Individuals Being Considered for Carbamazepine Therapy}

David S. Krause, MD, Kathleen Davis, BS, Daniel Dowd, PharmD and David J. Robbins, PhD

Genomind, Inc., King of Prussia, PA, USA

Presenting Author: David S. Krause

\begin{abstract}
Background. Carbamazepine, an anticonvulsant also used as a mood stabilizer and for trigeminal neuralgia, is associated with serious, sometimes fatal cutaneous adverse drug reactions, including Stevens Johnson Syndrome and toxic epidermal necrolysis1. Current literature demonstrates a genetic predisposition linked to specific class I and II human leukocyte antigen (HLA) types in various ethnic populations 2 . HLA-A ${ }^{\star} 31: 01$ is one such HLA type, and is routinely identified by the tag SNP rs1061235. However, rs1061235 has poor specificity for HLA*31:01 due to interference of HLA-A ${ }^{\star} 33$ types3. We investigated the false positive rate in our population and developed a novel real-time PCR assay that distinguishes HLA-A ${ }^{\star 31: 01}$ from other HLA-A types including HLA-A ${ }^{\star} 33$.

Methods. 120 unique samples were tested in triplicate during the validation of this assay and were sent to a reference lab for HLA next generation sequencing (NGS) typing, including 89 in-house samples and 31 Coriell samples with documented HLA typing results. The results from our real-time PCR assay were compared
\end{abstract}

